\title{
Results from the Daya Bay Reactor Neutrino Experiment
}

\author{
JUAN PEDRO OCHOA RICOUX*广 \\ LAWRENCE BERKELEY NATIONAL LABORATORY \\ E-mail: jpochoa@lbl.gov
}

\begin{abstract}
The precise determination of the mixing angle $\theta_{13}$ is one of the main priorities in the field of neutrino physics. This parameter is inextricably linked to the possibility of observing $\mathrm{CP}$ violation in the neutrino sector, which may in turn hold the key to other open questions in physics, such as the matter anti-matter asymmetry in the universe. The Daya Bay Reactor Neutrino Experiment has the highest sensitivity to this parameter among all the other experiments that are currently in operation or under construction. The experiment consists of multiple identical detectors placed underground at different distances from three groups of reactors, a configuration that minimizes systematic uncertainties and cosmogenic backgrounds. The most recent analysis with 139 days of data acquired in a six-detector configuration yields $\sin ^{2}\left(2 \theta_{13}\right)=0.089 \pm 0.010$ (stat. $) \pm 0.005$ (syst.). With a precision of $12.6 \%$, this is the world's most precise measurement on $\sin ^{2}\left(2 \theta_{13}\right)$ to date. The details of this rate-only analysis, as well as the current status and future prospects of the experiment, are reviewed here.
\end{abstract}

VIII International Workshop on the Dark Side of the Universe

June 10-15, 2012

Buzios, Rio de Janeiro, Brasil

\footnotetext{
*Speaker.

${ }^{\dagger}$ On behalf of the Daya Bay Collaboration.
} 


\section{Physics Motivation}

Neutrino physics is a field that has seen an explosion of activity in the last two decades due to its potential for uncovering many of nature's secrets. Not only are neutrinos a unique kind of elementary particle due to their exotic properties, but they also provide us with the first glimpse of physics beyond the Standard Model. Likewise, they play an important role in many cosmological processes. In fact, it might even be the case that the predominance of matter in our present universe is a consequence of $\mathrm{CP}$ violation in the neutrino sector.

One of the best handles for understanding these exotic particles is through the phenomenon of neutrino oscillation, whereby neutrinos change flavor as they travel. This phenomenon is a direct consequence of the fact that the neutrino weak eigenstates $\left(v_{\mathrm{e}}, v_{\mu}, v_{\tau}\right)$ are mixtures of the mass eigenstates $\left(v_{1}, v_{2}, v_{3}\right)$. This mixing is parameterized using the Pontecorvo-Maki-NakagawaSakata (PMNS) matrix $[1,2,3]$, which is typically parameterized in term of three mixing angles $\left(\theta_{12}, \theta_{13}, \theta_{23}\right)$ and a CP-violating phase $\delta$. Due to their quantum-mechanical nature, the probability of neutrinos oscillating at a given energy and baseline are not only functions of these four parameters, but also of the neutrino mass-squared differences $\Delta m_{32}^{2} \equiv m_{3}^{2}-m_{2}^{2}$ and $\Delta m_{21}^{2} \equiv m_{2}^{2}-m_{1}^{2}$.

The study of neutrino oscillations has recently made great strides and has entered an era of precision. For instance, $\Delta m_{32}^{2}$ and $\Delta m_{21}^{2}$ are now known to better than a few percent [4]. Moreover, this year has seen a quantum leap forward in our determination of the mixing angle $\theta_{13}$, for which only an upper limit [5] was known until just recently. The first indication of a non-zero value of $\theta_{13}$ was seen in the tension between the solar and long baseline reactor oscillation data [6]. Subsequent direct hints then arose from the long-baseline experiments T2K [7] and MINOS [8], and were followed by the results released by the Double-CHOOZ reactor experiment [9]. Shortly after, the Daya Bay Reactor Neutrino Experiment made an unambiguous measurement of a non-zero $\theta_{13}$ with a significance of more than five standard deviations [10]. These results were subsequently confirmed by the RENO Collaboration [11].

The precise determination of the $\theta_{13}$ mixing angle remains as one of the highest priorities in the field of neutrino physics. This angle is inextricably linked to the possibility of observing CPviolation in the leptonic sector, something that is of extreme interest in the community due to the above mentioned cosmological implications. Not only does a non-zero $\theta_{13}$ allow for the possibility of next generation oscillation experiments probing leptonic $\mathrm{CP}$-violation experimentally, but also of determining the neutrino mass hierarchy. A comparison between accelerator-based results and reactor-based results may in fact be useful in addressing some of these questions. Finally, this angle constitutes a key parameter of the Standard Model and is thus an important input to future extensions of it.

The most recent measurement of $\theta_{13}$ done by the Daya Bay collaboration, now published in [12], is presented here. A short review of the experiment is given first, followed by a presentation of the anti-neutrino selection employed and the oscillation analysis results. The current status and prospects of the experiment are then briefly addressed. 


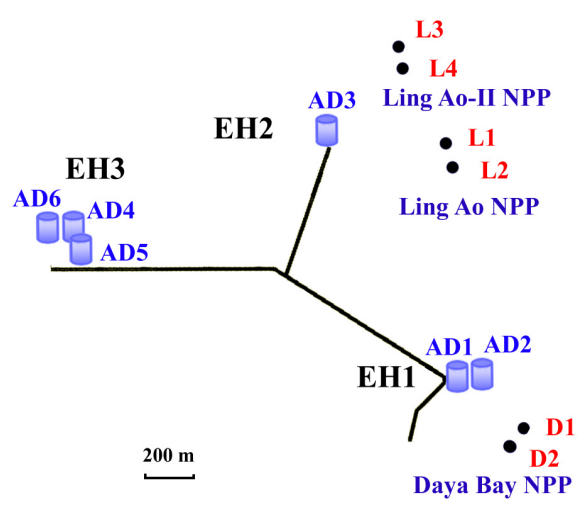

Figure 1: Schematic representing the layout of the Daya Bay Experiment at the time of the period considered for this analysis, where a total of six anti-neutrino detectors (represented as cylinders) had been deployed in the three experimental halls (EHs). The dots represent reactor cores, with D1-2 being the Daya Bay cores and L1-4 being the LingAo cores.

\section{The Daya Bay Experiment}

\subsection{Experimental Layout}

The Daya Bay nuclear power complex is located in the outskirts of the city of Shenzhen, China, approximately $55 \mathrm{~km}$ northeast of Hong Kong. It currently comprises a total of six nuclear reactors that make it one of the most powerful nuclear power plants in the world, with a total power output of $17.4 \mathrm{GW}_{\text {th }}$. The importance of this fact should not be underestimated as the sensitivity to $\theta_{13}$ depends directly on the magnitude of the neutrino flux that is sampled. Moreover, the Daya Bay complex is surrounded by mountains. This makes it easier to construct tunnels to reach underground laboratories with sufficient overburden to suppress backgrounds from cosmic rays.

The goal of the experiment is to accurately sample the electron anti-neutrino $\left(\bar{v}_{e}\right)$ flux produced by the nuclear reactors in two kinds of locations, one where the oscillation effects are very small (near), and another where the oscillation effects modulated by $\sin ^{2}\left(2 \theta_{13}\right)$ are maximal (far). The difference in measured flux between the two allows for the extraction of $\theta_{13}$. A schematic describing the layout of the experiment can be seen in Fig. 1. As seen there, the six reactors are spread out by more than one kilometer. Because of this, Daya Bay possesses the unique feature of having two near sites, allowing the experiment to obtain relative measurements of the contributions from the different cores and thus reduce the systematic uncertainties in the flux determination. The far site is located at a distance that is roughly equidistant from all the reactor cores and that maximizes the portion of the $\bar{v}_{e}$ disappearance probability that depends on $\sin ^{2}\left(2 \theta_{13}\right)$.

For the period corresponding to the analysis presented here only six detectors had been deployed, as illustrated in Fig. 1. This period elapsed from December 24th 2011 to May 11th 2012. As of October 19th 2012 however, a total of eight identical anti-neutrino detectors (ADs) have been acquiring data in the three experimental sites. Two detectors were deployed in each of the two near sites, while four were deployed in the far site. 

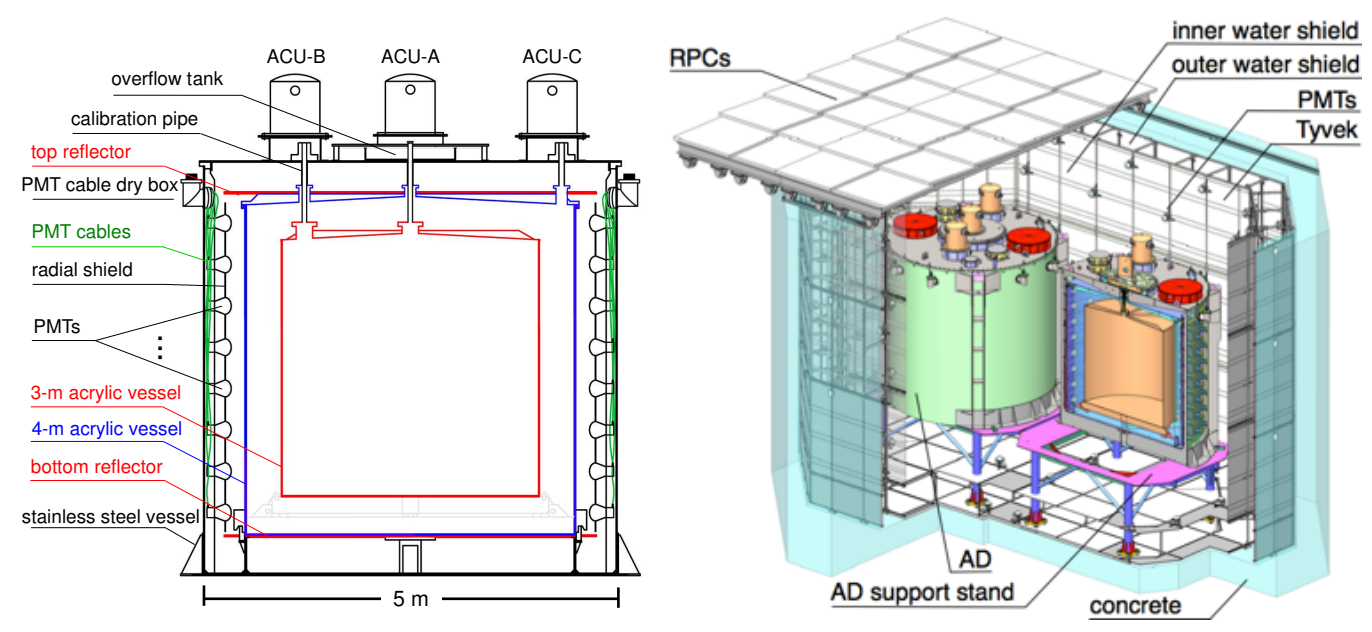

Figure 2: Cross-section of the anti-neutrino detectors (left) and of all the detector systems in a near site (right).

\subsection{Anti-neutrino detectors}

Anti-neutrinos are detected via the inverse beta-decay (IBD) reaction, given by

$$
\bar{v}_{e}+\mathrm{p} \longrightarrow \mathrm{e}^{+}+\mathrm{n} .
$$

As shown on the left side of Fig. 2, the detectors consist of three nested cylindrical volumes separated by concentric acrylic vessels, designed so that both of the products of this reaction are visible. The centermost zone, contained by the 3-m diameter acrylic vessel, holds 20 tons of gadoliniumloaded liquid-scintillator (Gd-LS) that serves as the target. There, most neutrons are captured in gadolinium nuclei roughly a fraction of a millisecond after the positron light is emitted:

$$
\mathrm{n}+\mathrm{Gd} \longrightarrow \mathrm{Gd}^{*} \longrightarrow \mathrm{Gd}+\gamma^{\prime} \mathrm{s}(8 \mathrm{MeV}) \text {. }
$$

By requiring a coincidence of the positron light with the delayed neutron capture light, the backgrounds can thus be greatly reduced. Surrounding the target, in the 4-m acrylic vessel, are another 20 tons of liquid-scintillator (LS), whose function is to avoid losing the gamma-ray energy for events that occur near the physical boundary of the target. Finally, an additional 40 tons of mineral oil shield the other two zones against radiation, which originates mostly from the wall of the tank and the 192 photomultiplier tubes (PMTs). Bottom and top reflectors are placed inside the tank to enhance the light collection.

Three automated calibration units (ACUs) are placed on the top of each anti-neutrino detector. Each ACU is able to deploy a light-emitting diode (LED) and three radioactive sources $\left({ }^{68} \mathrm{Ge},{ }^{60} \mathrm{Co}\right.$, ${ }^{241} \mathrm{Am}-{ }^{13} \mathrm{C}$ ) along the vertical axis of the detectors for calibration purposes. The detectors are assembled on the surface and filled underground. Their energy resolution is found to be $(7.5 / \sqrt{\mathrm{E}}+$ $0.9) \%$, where $\mathrm{E}$ is in $\mathrm{MeV}$ [13].

\subsection{The Muon Veto System}

The most important sources of background at Daya Bay are of cosmic-ray origin. Because of this, the detectors are submerged in instrumented water pools that serve a double purpose. First, 
the water is a good attenuator of gamma-rays from the ambient radioactivity as well as of neutrons produced by cosmic-rays. In fact, the pools are designed so that there are at least $2.5 \mathrm{~m}$ of water surrounding each $\mathrm{AD}$ in every direction. Second, the pools can function as Cerenkov detectors used for cosmic-ray muon tagging. As shown on the right of Fig. 2, in order to further increase the tagging efficiency, the pools are subdivided into two optically separated regions, the inner (IWS) and outer (OWS) water shields. In addition, a retractable roof of resistive plate chamber (RPC) modules is placed on top of the pools for increased background rejection. The water veto system and the RPC roof are designed to achieve a very high muon veto efficiency, larger than $(99.5 \pm 0.25) \%$. The RPC system was not used for the analysis presented here however.

\section{Anti-neutrino Event Sample}

\subsection{IBD Event Selection}

The dataset used in this analysis was acquired in a period spanning slightly less than six months, from December 24th 2011 to May 11th 2012. The IBD sample of events is obtained by searching for the coincidence of a positron-like event with a neutron-like event satisfying the following requirements: (i) the prompt-like event must have an energy between 0.7 and $12 \mathrm{MeV}$, (ii) the neutron-like event must have an energy between 6 and $12 \mathrm{MeV}$, and (iii) the time difference between these two must be between 1 and $200 \mu \mathrm{s}$. It should be noted that the AD trigger has almost $100 \%$ efficiency at $0.7 \mathrm{MeV}$, which is roughly three standard deviations away from the positron IBD threshold [13].

In addition, a multiplicity cut is applied in order to remove any ambiguities in the pair selection. This cut requires that no signals with $\mathrm{E}>0.7 \mathrm{MeV}$ occur $200 \mu$ s before the positron-like signal, $200 \mu \mathrm{s}$ after the neutron-like signal, and between them. Finally, a flasher rejection cut is also applied to ensure no events in which a PMT spontaneously emits light due to a discharge in its base are selected. Approximately 5\% of the PMTs exhibit this flashing behavior, where most of the light is typically concentrated around and directly in front of the flashing PMT. The details of this and all other cuts are in [12].

In order to suppress backgrounds associated with cosmic-rays, two types of muon veto cuts are also applied. First, neutron-like IBD candidates within a ( $-2 \mu \mathrm{s}, 600 \mu \mathrm{s})$ window with respect to a water shield muon event are rejected. A water shield muon event is defined as any signal where the number of PMTs hit in either the OWS or the IWS is larger than twelve. The lowerend of the cut window ( $-2 \mu \mathrm{s})$ safely accounts for any temporal non-synchronization among the different detectors. This cut removes most neutrons produced outside the AD, some of which could penetrate its walls. Second, a larger veto window is used for muons passing through the AD. For muon-like events, defined as events depositing more than $20 \mathrm{MeV}$ of energy in the $\mathrm{AD}$, the veto window is extended to $1 \mathrm{~ms}$. For showering muon-like events, defined as events depositing more than $2.5 \mathrm{GeV}$ of energy in the AD, it is extended to $1 \mathrm{~s}$. This second type of cut allows to greatly reduce most of the cosmogenic backgrounds produced by muons passing through the $\mathrm{AD}$.

With these cuts, the overall detection efficiency of IBD events is approximately $80 \%$, with the Gd-capture ratio $(\sim 84 \%)$ and the $6 \mathrm{MeV}$ energy cut for neutron-like events $(\sim 91 \%)$ being the dominant components [12]. 


\subsection{Backgrounds}

The accidental background, defined as any pair of otherwise uncorrelated signals that happen to satisfy the IBD selection criteria, is by far the largest contamination in the Daya Bay IBD sample. Its rate however can be accurately calculated through Poisson statistics given the measured rates of single positron-like events and single neutron-like events. This calculation yields an accidental background that amounts to about $1.7 \%$ of the near site IBD samples and to $4.6 \%$ of the far site sample. The corresponding error on these estimations is roughly $0.02 \%$ and $0.06 \%$ respectively.

The correlated $\beta-\mathrm{n}$ decays from cosmogenic ${ }^{9} \mathrm{Li} /{ }^{8} \mathrm{He}$ are the second largest contamination. Both of these elements, with respective lifetimes of $172 \mathrm{~ms}$ and $257 \mathrm{~ms}$, are produced from carbon nuclei as energetic cosmic muons pass through the liquid scintillator. The rate of correlated background from this source can be directly estimated by fitting the distribution of the time elapsed since the last muon with the known decay lifetimes. Such a procedure puts this contamination at $0.35 \%$ and $0.2 \%$ in the near and far sites respectively. A $50 \%$ relative systematic uncertainty is conservatively assumed due to the fitting procedure.

Another source of contamination is the correlated background from the Am-C neutron calibration sources. During data-taking the sources rest inside the ACUs on top of each AD, but neutrons emitted from them occasionally mimic IBD events. This happens through inelastic scattering with nuclei in the shielding material and the subsequent capture on a metal nuclei ( $\mathrm{Fe}, \mathrm{Cr}, \mathrm{Mn}$ or $\mathrm{Ni}$ ). This background is estimated through a GEANT4-based Monte Carlo simulation using the observed rate of single backgrounds from the Am-C sources. The resulting contamination is $0.03 \%$ at the near sites and $0.3 \%$ at the far site. A relative systematic uncertainty of $100 \%$ is conservatively assigned.

There is also the background caused by fast neutrons produced either inside or outside the muon veto system. Through elastic scattering with protons and the subsequent thermalization and capture on gadolinium, these neutrons can mimic the positron-like and neutron-like signals of IBD interactions. The energy of the proton recoil signal ranges from sub $\mathrm{MeV}$ to tens of $\mathrm{MeV}$, and it is thus possible to extrapolate the measured spectrum above $15 \mathrm{MeV}$ to lower energies. This estimate is cross-checked by selecting those fast neutrons whose primary muon was actually seen in the water shield. The resulting estimate for this contamination is $0.12 \%$ and $0.07 \%$ in the near and far sites respectively, with a conservative relative systematic uncertainty of approximately $30 \%$.

Finally, there is the background caused by the ${ }^{13} \mathrm{C}(\alpha, n){ }^{16} \mathrm{O}$ reaction brought about by natural radioactivity. With the aid of a Monte Carlo simulation, supplemented with the measured alphadecay rates from ${ }^{210} \mathrm{Po},{ }^{238} \mathrm{U},{ }^{232} \mathrm{Th}$ and ${ }^{227} \mathrm{Ac}$, it is possible to estimate this background. The resulting contamination estimates are $0.01 \%$ and $0.05 \%$ in the near and far sites respectively, with a $50 \%$ relative systematic uncertainty.

\section{Oscillation Analysis}

For the oscillation analysis the prediction of the expected signal at the far site is made using the information from the two near hall measurements. Because of this, any correlated detector and/or reactor uncertainties have a negligible impact on the result.

The largest uncorrelated detector-related uncertainty is associated with the $6 \mathrm{MeV}$ cut for neutron-like events, and amounts to $0.12 \%$. The next largest uncertainty originates from the relative 


\begin{tabular}{c|c|c|c|c|c}
$\mathrm{AD} 1$ & $\mathrm{AD} 2$ & $\mathrm{AD} 3$ & $\mathrm{AD} 4$ & $\mathrm{AD} 5$ & $\mathrm{AD6}$ \\
\hline $662.47 \pm 3.00$ & $670.87 \pm 3.01$ & $613.53 \pm 2.69$ & $77.57 \pm 0.85$ & $76.62 \pm 0.85$ & $74.97 \pm 0.84$
\end{tabular}

Table 1: Measured anti-neutrino interaction rates in all six detectors after corrections for livetime, veto efficiencies and background.

differences in neutron-Gd capture ratios, and is conservatively estimated at $0.1 \%$. Other detectorrelated uncertainties, such as the error in the determination of the number of target protons, are quite small in comparison. A detailed summary can be found in [12]. The combination of all uncorrelated detector-related uncertainties amounts to $0.2 \%$.

Similarly, the uncorrelated reactor-related uncertainties include a $0.5 \%$ error assigned to the thermal power data and a $0.6 \%$ error to the fission fraction information that is received from the power plant. A $0.3 \%$ systematic uncertainty is also assigned to the anti-neutrinos produced from spent fuel. This yields a total uncorrelated reactor uncertainty of $0.8 \%$, which is ultimately reduced in the analysis due to the near/far comparison. The choice of flux model has a negligible impact on the results.

The observed anti-neutrino rates per day, after all corrections due to livetime, veto efficiencies, and backgrounds have been applied, are given in table 1 . The small differences observed for sideby-side detectors are consistent with the fact that they are in slightly different locations. The ratio of the observed to the expected number of events in the far site, where the latter is calculated from the observed rate at the near sites in the assumption of no oscillations, yields a result of $R=0.944 \pm 0.007$ (stat.) \pm 0.003 (syst.).

A chi-square method with penalty terms is used to extract $\sin ^{2}\left(2 \theta_{13}\right)$ based on the standard 3flavor oscillation probability. Care was taken to ensure the that the uncorrelated uncertainties from the backgrounds, detectors, reactor fluxes and oscillation parameters were properly accounted for. The left panel of Fig. 3 shows the number of IBD candidates in each detector after all corrections, relative to those expected assuming no oscillations. The absolute normalization was determined from the fit to the data.

The best-fit value obtained in this analysis is

$$
\sin ^{2}\left(2 \theta_{13}\right)=0.089 \pm 0.010 \text { (stat.) } \pm 0.005 \text { (syst.), }
$$

which excludes the no-oscillation hypothesis at 7.7 standard deviations. The right panel of Fig. 3 shows the observed positron spectrum in the far site compared to the near site measurements. The spectral distortion observed is consistent with the oscillation hypothesis at the best-fit $\theta_{13}$.

\section{Conclusion and Outlook}

With a precision of $12.6 \%$ in $\sin ^{2}\left(2 \theta_{13}\right)$, the result reported here constitutes the world's most precise measurement of this quantity to date. It is consistent with and supersedes the previous result [10]. Currently, the total uncertainty on $\theta_{13}$ is still largely dominated by the statistical uncertainty. With the full eight-AD configuration however, which began physics data-taking in October of this year, we expect to achieve a $\sim 5 \%$ measurement of $\sin ^{2}\left(2 \theta_{13}\right)$ in a period of about three years. Moreover, the Daya Bay experiment is also sensitive to the effective squared-mass difference $\Delta m_{e e}^{2}$, which is a combination of $\Delta m_{31}^{2}$ and $\Delta m_{32}^{2}$. Finally, the high statistics IBD samples 

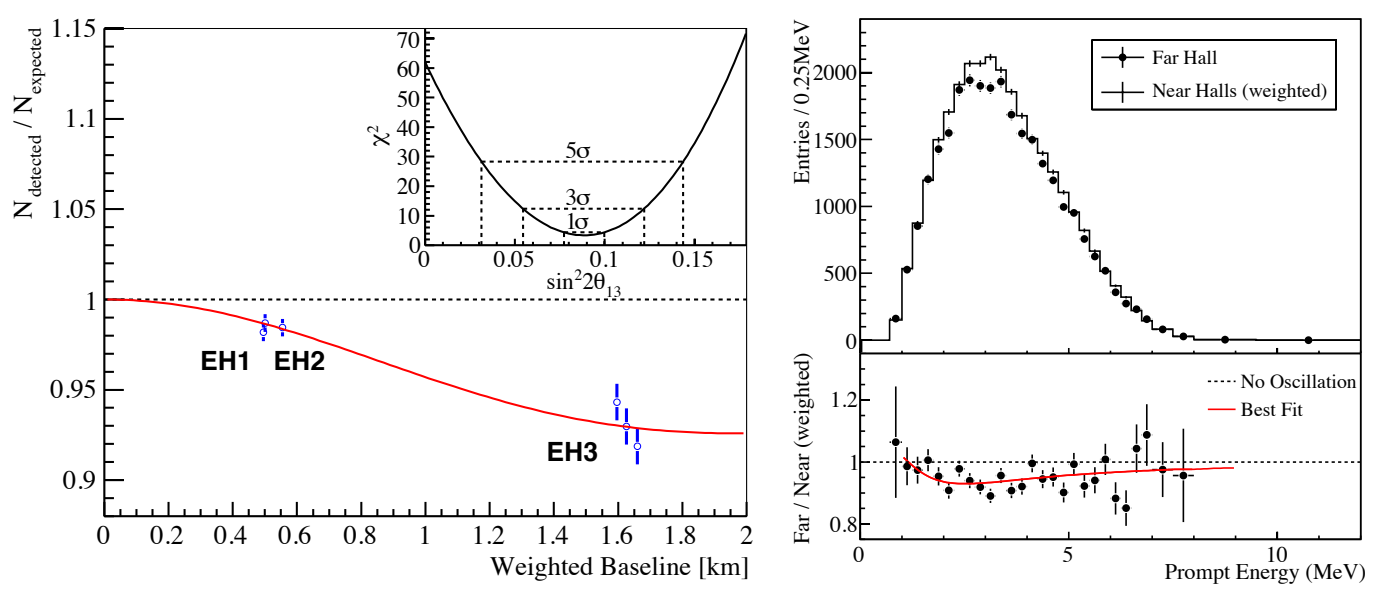

Figure 3: Left: ratio of measured versus expected anti-neutrino interactions with the no oscillations assumption, as well as the $\chi^{2}$ value versus $\sin ^{2}\left(2 \theta_{13}\right)$. The oscillation survival probability at the best-fit value is given by the smooth curve, and the AD4 and AD6 data points were displaced by $\pm 30 \mathrm{~m}$ for visual clarity. The error bars quantify the total uncorrelated uncertainty of each AD. Right: measured prompt energy spectrum at the far site (three ADs combined) compared with the no-oscillation prediction based on the near site measurements. Errors are statistical only. The bottom panel shows the ratio of the measured vs. the expected spectra.

from Daya Bay can yield the world's most precise measurement of the anti-neutrino energy spectrum, something that is essential to future reactor experiments. Results in these areas can thus be expected in the future.

\section{References}

[1] B. Pontecorvo, Sov. Phys. JETP 6, 429 (1957).

[2] B. Pontecorvo, Sov. Phys. JETP 26, 984 (1968).

[3] Z. Maki, M. Nakagawa, and S. Sakata, Prog. Theor. Phys. 28, 870 (1962).

[4] J. Beringer et al. (Particle Data Group), Phys. Rev. D86, 010001 (2012).

[5] M. Apollonio et al. (CHOOZ Collaboration), Eur. Phys. J. C27, 331 (2003).

[6] G. Fogli et al., Phys. Rev. Lett. 101, 141801 (2008).

[7] K. Abe et al. (T2K Collaboration), Phys. Rev. Lett. 107, 041801 (2011).

[8] P. Adamson et al. (MINOS Collaboration), Phys. Rev. Lett. 107, 181802 (2011).

[9] Y. Abe et al. (Double Chooz Collaboration), Phys. Rev. Lett. 108, 131801 (2012).

[10] F.P. An et al. (Daya Bay Collaboration), Phys. Rev. Lett. 108, 171803 (2012).

[11] J.K. Ahn et al. (RENO Collaboration), Phys. Rev. Lett. 108, 191802 (2012).

[12] F.P. An et al. (Daya Bay Collaboration), Chinese Physics C37, 011001 (2013).

[13] F.P. An et al. (Daya Bay Collaboration), Nucl. Inst. Meth. A685, 78 (2012). 